\title{
Bacopa monnieri as augmentation therapy in the treatment of anhedonia, preclinical and clinical evaluation
}

\author{
Laura Micheli $^{1}$ | Silvia Spitoni ${ }^{2}$ | Lorenzo Di Cesare Mannelli $^{1} \odot$ | Anna Rita Bilia ${ }^{3}$ | \\ Carla Ghelardini $^{1}$ | Stefano Pallanti ${ }^{2,4,5}$
}

\author{
${ }^{1}$ Department of Neuroscience, Psychology, \\ Drug Research and Child Health- \\ NEUROFARBA-Pharmacology and \\ Toxicology Section, University of Florence, \\ Florence, Italy \\ ${ }^{2}$ Department Neurofarba, Psychiatry Section, \\ University of Florence, Florence, Italy \\ 3Department of Chemistry "Ugo Schiff", \\ University of Florence, Florence, Italy \\ ${ }^{4}$ Department of Psychiatry and Behavioral \\ Sciences, Albert Einstein College of Medicine, \\ New York, New York \\ ${ }^{5}$ Institute of Neuroscience, Florence, Italy \\ Correspondence \\ Lorenzo Di Cesare Mannelli, Department of \\ Neuroscience, Psychology, Drug Research and \\ Child Health-NEUROFARBA-Pharmacology \\ and Toxicology Section, University of \\ Florence, Viale Pieraccini 6, Florence 50139, \\ Italy. \\ Email: lorenzo.mannelli@unifi.it
}

Bacopa monnieri (L.) is widely used in Ayurvedic medicine as a neural tonic for improving intelligence and memory. Several studies highlighted its efficacy in neuropsychiatric diseases but there is no evidence regarding anhedonia. Aim of the present work was to preclinically and clinically test against anhedonia a standardized B. monnieri extract ( $20 \%$ bacosides). In a mouse model of a depressive-like syndrome induced by lipopolysaccharide (LPS), the daily administration of the extract (50$200 \mathrm{mg} \mathrm{kg}^{-1}$, p.o.) for 1 week, dose-dependently counteracted the immobility time in Porsolt and Tail suspension tests $(p<.01)$. At the sucrose preference test (directly related to the ability for feeling pleasure) the extract treatment (100 and $200 \mathrm{mg} \mathrm{kg}^{-1}$ ) counteracted the reduction of sucrose intake induced by LPS $(p<.01)$. Moreover, B. monnieri significantly reduced cytokines, cortisol, and artemin LPS-dependent alterations in plasma while increased the brain-derived neurotrophic factor levels $(p<.05)$. The efficacy of the same extract was tested in a clinical study in which 42 patients with significant degree of anhedonia (evaluated as Snaith-Hamilton Pleasure Scale [SHAPS] score $\geq 3$ ) were enrolled. Patients were divided into two groups and treated with citalopram or citalopram associated with B. monnieri $(300 \mathrm{mg}$ bid) for 4 weeks. The Pears Sample T-test showed a significant improvement $(p<.05)$ in relevant scales (Hamilton depression rating scale, SHAPS, and strength and difficulties questionnaire) in the extract-treated group in comparison to citalopram alone was recorded. These data suggest that $B$. monnieri extract may be effective for the management of anhedonia and therefore should be considered for future controlled trials.

\section{KEYWORDS}

artemin, BDNF, HAM-D, LPS, SDQ, SHAPS
Abbreviations: ARTN, artemin; BDNF, brain-derived neurotrophic factor; CEQ, credibility/ expectancy questionnaire; HAM-D, Hamilton depression rating scale; LPS, bacterial lipopolysaccharide; RDoC, Research Domain Criteria; SDQ, strength and difficulties questionnaire; SHAPS, Snaith-Hamilton Pleasure Scale; TAU, conventional therapy; TBARS, thiobarbituric acid reactive substances.

Laura Micheli, Silvia Spitoni, Carla Ghelardini, and Stefano Pallanti contributed equally to this manuscript.

\section{1 | INTRODUCTION}

Bacopa monnieri, a plant native to the wetlands of Southern and Eastern India, is known in India under the name of Brahmi and is traditionally used in Ayurvedic medicine as neural tonic, sedative, anti-epileptic, memory, and learning enhancer (Pham et al., 2019). Several preclinical studies have shown numerous pharmacological effects of this plant, such as improved memory in Alzheimer's disease and schizophrenia, potential anti-stress, anticonvulsant and anti-Parkinson's actions and recent clinical trials have 
confirmed the efficacy of $B$. monnieri on learning and cognitive performance (Negi, Singh, \& Kushwaha, 2000). Furthermore, numerous preclinical studies have demonstrated the antidepressant effects of a $B$. monnieri standardized extracts in paradigms commonly used to evaluate depression (Banerjee, Hazra, Ghosh, \& Mondal, 2014; Sheikh et al., 2007). Moreover, B. monnieri can inhibit the release of IL- 6 and TNF- $\alpha$ from activated microglia (Nemetchek, Stierle, Stierle, \& Lurie, 2017) and increase concentration of the brain-derived neurotrophic factor (BDNF) in rat hippocampus (Banerjee et al., 2014; Hazra, Kumar, Saha, \& Mondal, 2017; Kumar \& Mondal, 2016). Lesser evidence exist about the clinical efficacy of B. monnieri, a double-blind RCT study on mood and stress (Benson et al., 2014) was carried out on 17 healthy volunteers treated with 320 and $640 \mathrm{mg}$ of extract after being exposed to stress induced by multitasking: the results showed a cortisol levels reduction and mood improvement (Benson et al., 2014).

Depression may be intended as the expression of different endophenotypes (Hasler, Drevets, Manji, \& Charney, 2004). The association between psychopathological (impaired reward and cognitive functions, neurovegetative signs, increased stress sensitivity) and biological (functional and structural brain abnormalities, dysfunctions in neurotransmitters levels, and intracellular signaling) endophenotypes generates multiplex behavioral manifestations related to different neural circuits according to the Research Domain Criteria (RDoC) model (Insel, 2014). Beyond heterogeneity, anhedonia, the reduced ability to experience pleasure, is a recurrent, transnosographic dimension. It is a hallmark symptom of major depression, schizophrenia, and other neuropsychiatric disorders that represents a negative prognostic factor and leads to adverse mental health outcomes across the lifespan and predicts poor psychosocial functioning. The neural bases of the construct of anhedonia reflects deficits in hedonic capacity and are closely linked to the constructs of reward valuation, decision-making, anticipation, and motivation (Treadway \& Zald, 2011). Since anhedonia is a difficult-to-treat target, several therapeutic approaches have been proposed, including psychosocial interventions, antipsychotics (for schizophrenia conditions) (Aleman et al., 2017), antidepressants (in mood disorders) (Craske, Meuret, Ritz, Treanor, \& Dour, 2016), and neuromodulation interventions (in addictive use disorder; Pettorruso et al., 2018; Pettorruso, Di Giannantonio, De Risio, Martinotti, \& Koob, 2019).

On this base, the possibility to intervene on the anhedonic dimension with a natural complementary approach becomes an intriguing therapeutic possibility. B. monnieri is a theoretically optimal candidate to be evaluated. Aim of the present work was to study the efficacy of a characterized B. monnieri extract in a mouse model of LPS-induced anhedonia and, in the subsequent clinical phase of the study, to test the effect of the extract in addition to conventional therapy (TAU) in patients with this symptom.

\section{2 | MATERIALS AND METHODS}

\section{1 | Extract and pure Bacoside II}

A commercial B. monnieri powder extract (Galeno, Carmignano, Italy) was used for the tests, batch n. C17070624. Bacoside II was an international reference standard (purity 98\%) from Abacipharm Corp (Maryland, USA).

\section{2 | HPLC-DAD and ESI-TQ-MS/MS apparatus and conditions}

The HPLC analysis was performed on a HPLC 2695 Waters instrument coupled with TQ-Four micro Waters using an ESI source. The chromatographic separation was performed using a Gemini C18 analytical column $150 \times 2.0 \mathrm{~mm}(5 \mu \mathrm{m}$, particle size, $110 \AA$ ) Phenomenex. The flow rate was $0.35 \mathrm{ml} / \mathrm{min}$ and the injection volume was $20 \mu \mathrm{l}$. Mobile phase (water/acetonitrile) was a gradient water starting with $20 \%$ of acetonitrile until $80 \%$ in 23 min. Identification of peaks was based on comparisons of ESI-MS/MS spectra with those reported in the literature and co-injection with standard Bacoside II. Samples were dissolved in $\mathrm{MeOH}(1 \mathrm{mg} / \mathrm{ml})$ and filtered in a $0.45 \mu \mathrm{m}$ membrane filter. Acquisition in full scan MS, positive ion mode from $\mathrm{m} / \mathrm{z}$ 430 to $\mathrm{m} / \mathrm{z}$ 1,200. The quantification of the contents of the B. monnieri saponins was performed using HPLC-MS with external standard method, using Bacoside II (IS, 99.7\% pure by HPLC) as standard compound. The HPLC method was validated in terms of linearity, limit of detection, limit of quantification, precision, accuracy, and robustness according to the International Conference on Harmonization (ICH) guidelines (ICH, 2005).

\section{3 | Preclinical investigation}

\subsection{1 | Animals}

Male CD-1 albino mice (Envigo, Varese, Italy) weighing approximately 22-25 g at the beginning of the experimental procedure, were used. Animals were housed in CeSAL (Centro Stabulazione Animali da Laboratorio, University of Florence) and used at least 1 week after their arrival. A maximum of 12 mice were housed per cage (size $26 \times 41 \mathrm{~cm}$ ); animals were fed a standard laboratory diet and tap water ad libitum, and kept at $23 \pm 1^{\circ} \mathrm{C}$ with a $12 \mathrm{~h}$ light/dark cycle, light at 7 a.m. All animal manipulations were carried out according to the Directive 2010/63/EU of the European parliament and of the European Union council (September 22, 2010) on the protection of animals used for scientific purposes. The ethical policy of the University of Florence complies with the Guide for the Care and Use of Laboratory Animals of the US National Institutes of Health (NIH Publication No. 85-23, revised 1996; University of Florence assurance number: A5278-01). Formal approval to conduct the experiments described was obtained from the Italian Ministry of Health (No. 54/2014-B) and from the Animal Subjects Review Board of the University of Florence. Experiments involving animals have been reported according to ARRIVE guidelines (McGrath \& Lilley, 2015). All efforts were made to minimize animal suffering and to reduce the number of animals used. 


\subsection{2 | B. monnieri treatment}

The extract of B. monnieri (L.) Pennell (dry extract, 20\% bacosides, Galeno Srl, Italy) was suspended in $1 \%$ carboxymethylcellulose sodium salt (CMC) and orally (p.o.) administered every day for 1 week in a range dose of $50-200 \mathrm{mg} \mathrm{kg}^{-1}$. Control group was treated with vehicle.

\subsection{3 | LPS-induced anhedonia}

Lipopolysaccharide (LPS) from Escherichia coli was purchased from Sigma-Aldrich, freshly dissolved in sterile saline and injected intraperitoneally (i.p.) at the dose of $1.25 \mathrm{mg} \mathrm{kg}^{-1}$ (Biesmans et al., 2013) after 1 week of $B$. monnieri treatment, $1 \mathrm{hr}$ after the last administration of the extract. Behavioral tests were performed before 6 and $24 \mathrm{hr}$ after LPS administration. Control group was treated with vehicle.

\subsection{4 | Forced swimming test}

The forced swimming test used was the same described by Porsolt, Le Pichon, and Jalfre (1977). More information are reported in Supporting Information.

\subsection{5 | Tail suspension test}

A piece of tape was adhered to the upper middle of the tail of each animal, creating a flap with the overlap of tape. Mice were suspended from a plastic rod mounted $50 \mathrm{~cm}$ above the surface by fastening the tail to the rod with adhesive tape. The duration of the test was 6 min and the immobility time was measured in the first $2 \mathrm{~min}$, when animals react to the inescapable stress, and in the last 4 min of the test, when the behavioral despair is established. Immobility was defined as the absence of any limb or body movements, except those caused by respiration.

\subsection{6 | Sucrose preference}

The preference for $2 \%$ sucrose solution was examined as a measured for anhedonia. More information are reported in Supporting Information.

\subsection{7 | Tissue collection and measurements of soluble factors}

At the end of the behavioral test session, $24 \mathrm{hr}$ after LPS administration, animals were sacrificed by decapitation, the prefrontal cortex was collected and frozen using liquid nitrogen. IL-6, IL-1 $\beta$, cortisol, BDNF, and ART were detected using ELISA kit according to the manufacturer instructions.

\subsection{8 | Determination of thiobarbituric acid reactive substances}

Thiobarbituric acid reactive substances (TBARS) were quantified in brain tissue homogenates as described previously, with some minor modifications (Pan et al., 2009).

About $100 \mu \mathrm{g}$ of prefrontal cortex tissue homogenate were added to $4 \mathrm{ml}$ reaction mixture consisting of $36 \mathrm{mM}$ thiobarbituric acid (Sigma-Aldrich, Milan, Italy) solubilized in $10 \% \mathrm{CH}_{3} \mathrm{COOH}$, $0.2 \%$ SDS, $\mathrm{pH}$ was adjusted to 4.0 with $\mathrm{NaOH}$. The mixture was heated for $60 \mathrm{~min}$ at $100^{\circ} \mathrm{C}$ and the reaction was stopped by placing the vials in ice bath for $10 \mathrm{~min}$. After centrifugation (at $1,600 \mathrm{~g}$ at $4^{\circ} \mathrm{C}$ for $10 \mathrm{~min}$ ) the absorbance of the supernatant was measured at $532 \mathrm{~nm}$ (Perkin-Elmer spectrometer, Monza, Italy) and TBARS were quantified in $\mu$ moles/milligram of total protein using 1,1,3,3-tetramethoxypropane as standard. Protein homogenate concentration was measured by bicinchoninic acid (BCA; SigmaAldrich) assay.

\subsection{9 | Catalase activity}

Catalase activity was measured in the supernatant of brain tissue homogenate by Amplex Red Catalase Assay Kit (Invitrogen, Monza, Italy) following the manufacturer's instructions as previously described (Zanardelli et al., 2014). Protein concentration was quantified by bicinchoninic acid assay (Sigma-Aldrich). Catalase activity for each sample was normalized to protein concentration. Control conditions in the absence of treatment were set as $100 \%$.

\subsection{Clinical investigation}

\subsection{1 | Sample}

Our study was conducted on a sample of patients described in Table 1, dealing with outpatients and Day Hospital of the Psychiatric unit in the University Hospital of Florence and Institute of Neuroscience (INS), which had a clinically significant degree of anhedonia (evaluated as SHAPS score $\geq 3$ ) being diagnosed for major depression (DSM 5.0) and after adequate treatment with citalopram and stable for 4 weeks with an unsatisfactory response.

\subsection{2 | Assessment}

To measure the severity of anhedonia, every participant was administered the Snaith-Hamilton Pleasure Scale (SHAPS; Snaith et al., 1995) and the Temporal Experience of Pleasure Scales (TEPS) scale immediately before and after the 4-week treatment period. Moreover, the following scales were administered and evaluated: strengths and difficulties questionnaire (SDQ), patient health questionnaire, Hamilton 
TABLE 1 Study of the sample

\begin{tabular}{|c|c|}
\hline Sample & 42 Patients, 19 cases (BM), and 23 controls (TAU) \\
\hline Sex & $F>M(64.3 \% N=27$ vs. $35.7 \% N=15)$ \\
\hline Age & $\begin{array}{l}\text { In cases of average age } 47.22 \text { years (DS }=12.891) \text {, } \\
\text { between checks } 48.53 \text { years (DS = 13.443) } \\
\text { For the years of illness, the two groups show no } \\
\text { statistically significant differences }(p<.05) \text { : The } \\
\text { cases have average disease duration of } \\
15.55 \text { years (DS }=10.828 \text { ) and the controls of } \\
8.76 \text { years (DS }=11.098 \text { ) }\end{array}$ \\
\hline $\begin{array}{l}\text { Average } \\
\text { school age }\end{array}$ & 12.47 years (DS 3.31) \\
\hline Smoke & $21 \%$ smokers, $21.4 \%$ N = 9 smokers \\
\hline Job & $\begin{array}{l}\text { Employees } 59.5 \% N=25 \text {, freelancers } 9 \% N=5 \text {, } \\
\text { retired } 16.7 \% N=7 \text {, unemployed } 11.9 \% N=5\end{array}$ \\
\hline
\end{tabular}

depression rating scale (HAM-D; Hamilton et al., 1960), credibilityexpectation questionnaire (CEQ), and clinical global impression (CGI).

\subsection{3 | Treatment}

Patients were randomly assigned to two groups. The first group ( $n=23$ ) was treated only with $(40 \mathrm{mg}$ ) citalopram for the follow 4 weeks (TAU); the second group $(n=19)$ with conventional therapy associated with a nutraceutical supplement consisting of B. monnieri extract titrated at $20 \%$ in bacosides (one capsule $300 \mathrm{mg}$ twice daily). The treatment lasted 4 weeks.

\subsection{4 | Statistical analysis}

For preclinical study, behavioral measurements were performed on 16 mice for each treatment carried out in two different experimental sets. Results were expressed as means \pm SEM and the analysis of variance was performed by ANOVA. A Bonferroni's significant difference procedure was used as post-hoc comparison. $p$ Values of less than 05 and .01 were considered significant. Data were analyzed using the "Origin 8.1" software. For clinical study, in order to characterize the study sample, a descriptive statistical analysis was performed. In particular for the categorical variables study the chi-square test was used, while the $t$-test was used for the continuous variables. For some variables, in addition to the paired $t$-test, a nonparametric test; a multivariate linear model was therefore used to study the association between the use of the bacopa and the outcome of the tests and to correct for the baseline CIB-3 imbalance.

Pearson's $r$ coefficient was calculated to evaluate the correlation of clinical variables.

Statistical tests were two-tailed. Level of significance was set at $p=.05$.

All analyses were performed on study completers and were carried out using the SPSS statistical software version 15.0 (SPSS, Inc., Chicago, 2007).

\section{3 | RESULTS}

\subsection{Characterization of the commercial extract}

B. monnieri commercial extract was characterized by HPLC-PDA-MS analysis. The chromatogram of $B$. monnieri commercial extract solubilized in $\mathrm{MeOH}(100 \mathrm{ng} / \mu \mathrm{l})$ displayed four principal peaks related to Bacopa saponins with retention times of 16.5, 16.8, 17.2, and $17.5 \mathrm{~min}$. The identification of Bacopaside II with the peak having a retention time of $16.8 \mathrm{~min}$ was based on the co-elution with the reference standard ( $20 \mathrm{ng} / \mu \mathrm{l}$ in methanol) and confirmed by the $\mathrm{m} / \mathrm{z} 951.8$ of the $[\mathrm{M}+\mathrm{Na}]^{+}$.

The other three peaks of the chromatogram of B. monnieri commercial extract at $16.5,17.2$, and 17.5 min were unambiguously attributed to Bacoside A3, Bacopasaponin C, and Bacopaside X, respectively. Peak at 16.5 was assigned to Bacoside $A 3$, with the same MS data of Bacopaside II, which represents its isomer. Bacopasaponin $C$ and Bacopaside $X$ were assigned to the peaks at 17.2 and $17.5 \mathrm{~min}$ because the characteristic MS data $(\mathrm{m} / \mathrm{z} 921.8$ or 921.9 of the [M $+\mathrm{Na}]+$ ), as reported in Figure 1 .

The repeatability was determined by HPLC analysis of three samples, and the RSD of the contents was calculated for the four major constituents. RSD values ranged between 0.55 and 3.80. Lastly, the external standard method was applied to quantify each compound, using a regression curve, with each point determined in triplicate. The resulting percentage of total saponins expressed as Bacopaside II was ca. $20 \%$.

\section{2 | Preclinical results}

\subsection{1 $\quad$ Forced swimming test}

We evaluated the protective effect of B. monnieri extract on LPSinduced anhedonia in the mouse. In the forced swimming test, behavioral despair was evaluated by measuring the time during which the animal remains immobile after being placed in a water filled cylinder from which it cannot escape (Figure 2). LPS (1.25 mg kg ${ }^{-1}$ ) acute administration was able to significantly increase the immobility time of the animals in this test both at 6 and $24 \mathrm{hr}$ after injection with respect to the control group (168.0 $\pm 13.7 \mathrm{~s}$ vs. $63.5 \pm 9.2 \mathrm{~s}$ at $6 \mathrm{hr}$ and $104.8 \pm 14.9 \mathrm{~s}$ vs. $40.0 \pm 7.9 \mathrm{~s}$ at $24 \mathrm{hr}$, respectively). Daily administration of $B$. monnieri extract for 1 week (50-200 $\mathrm{mg} \mathrm{kg}^{-1}$ ) was able to counteract the immobility time induced by LPS in a dose-dependent manner (Figure 2). The higher dose $\left(200 \mathrm{mg} \mathrm{kg}^{-1}\right)$ significantly diminished the reduction of movements induced by LPS systemic injection at 6 and $24 \mathrm{hr}$ (30.6 $\pm 17.4 \mathrm{~s}$ and $31.2 \pm 7.2 \mathrm{~s}$, respectively). The dose of $100 \mathrm{mg} \mathrm{kg}^{-1}$ was still active at both time points but with a lower efficacy $(70.3$ $\pm 21.0 \mathrm{~s}$ and $61.0 \pm 7.6 \mathrm{~s}$, respectively) while the dose of $50 \mathrm{mg} \mathrm{kg}^{-1}$ was inactive both at $6 \mathrm{hr}$ than $24 \mathrm{hr}(156.3 \pm 36.4 \mathrm{~s}$ and $95.7 \pm 8.7 \mathrm{~s}$, respectively). 

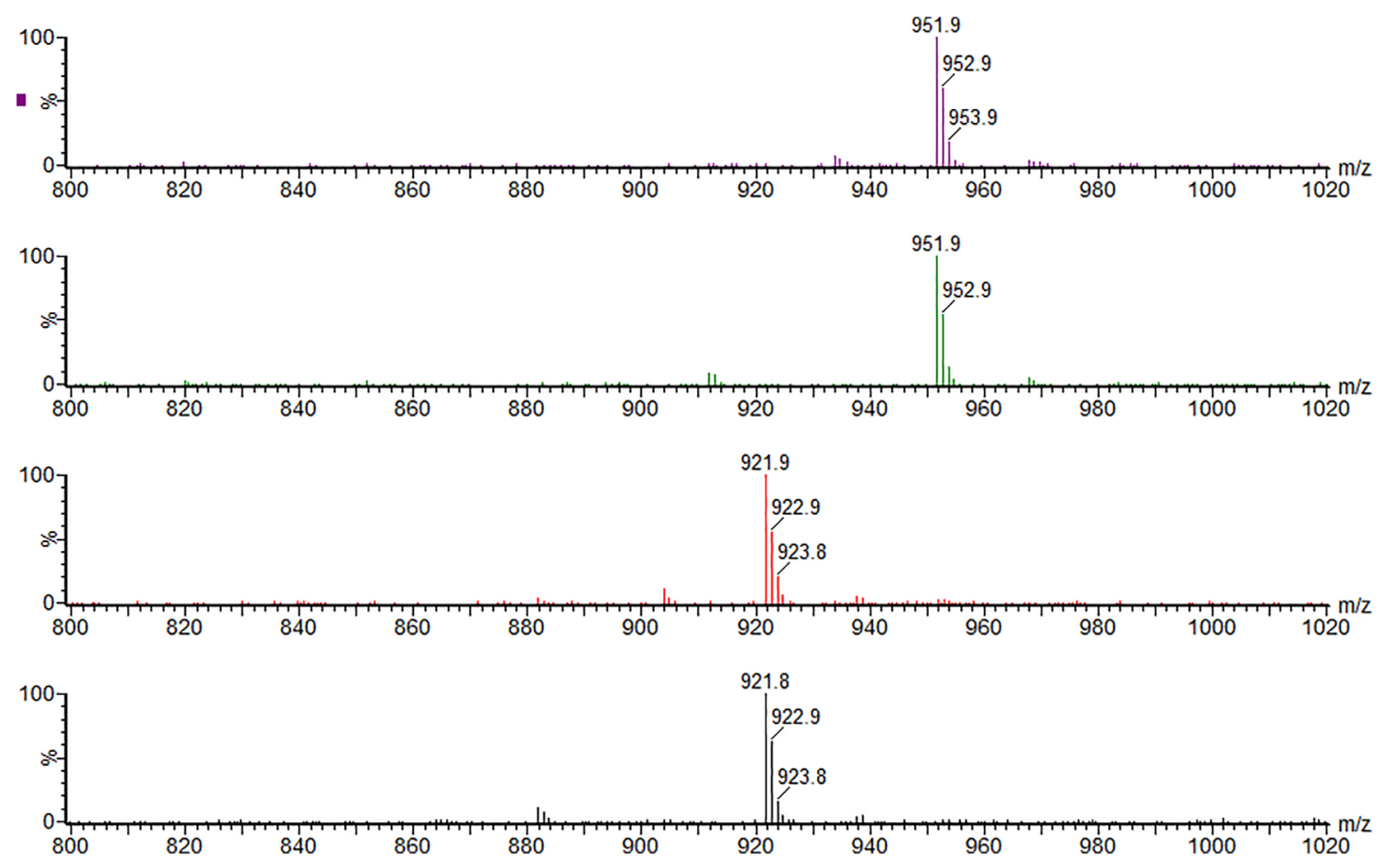

FIGURE 1 MS spectra of the positive ions of the major four peaks corresponding to the four detected saponins [Colour figure can be viewed at wileyonlinelibrary.com]

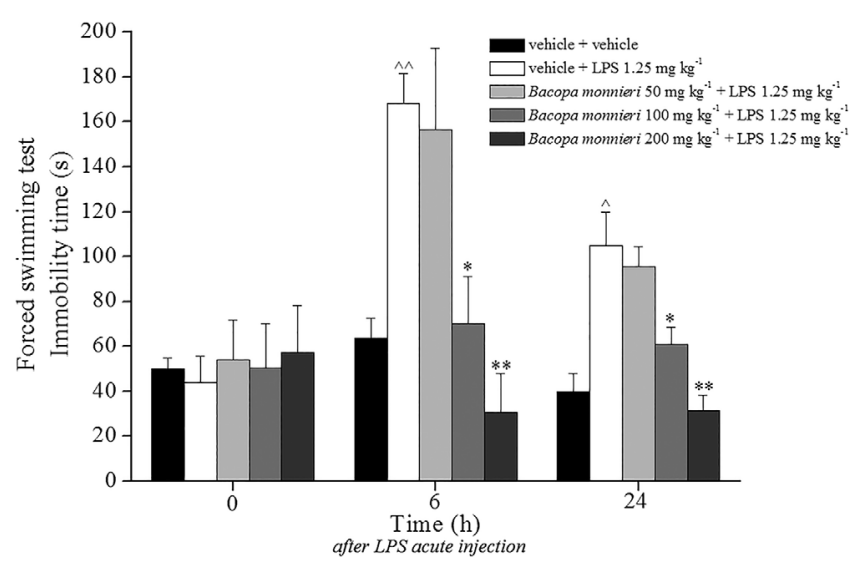

FIGURE 2 Forced swimming test. Effect of B. monnieri extract on LPS-induced anhedonic-like behavior. B. monnieri (50-200 mg kg-1) was p.o. daily administered for 1 week before the acute i.p. injection of LPS (1.25 mg kg-1). The immobility time was evaluated before and after LPS administration ( 6 and $24 \mathrm{hr}$ ). Each value represents the mean of 16 mice performed in two different experimental sets. Data are shown as mean $\pm S E M ; \wedge p<.05$ and ${ }^{\wedge} p<.01$ versus vehicle + vehicle-treated mice; ${ }^{*} p<.05$ and ${ }^{* *} p<.01$ versus vehicle + LPStreated mice. LPS, lipopolysaccharide

\subsection{2 | Tail suspension test}

Similar results were obtained in the tail suspension test (Figure 3). In this test, behavioral despair was evaluated by measuring the time during which the animal remains immobile after being suspended by the

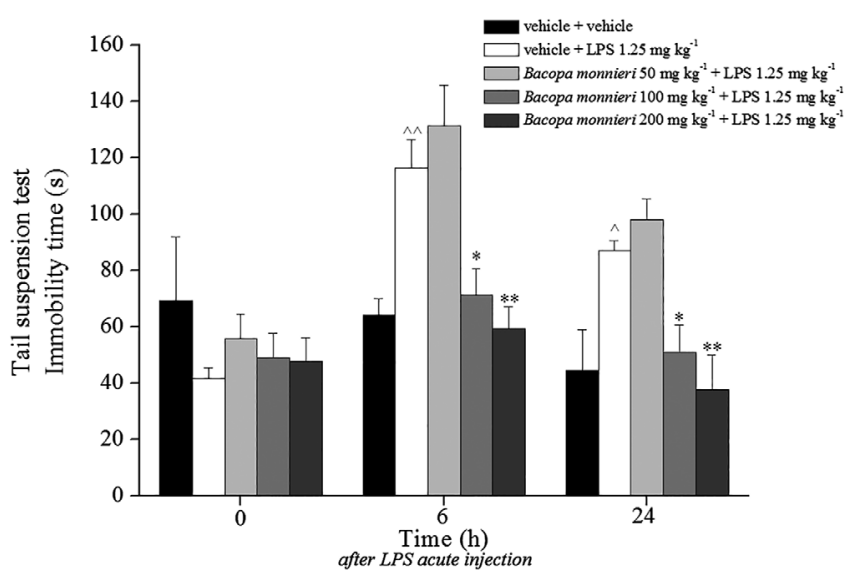

FIGURE 3 Tail suspension test. Effect of B. monnieri extract on LPS-induced anhedonic-like behavior. B. monnieri (50-200 mg kg-1) was p.o. daily administered for 1 week before the acute i.p. injection of LPS (1.25 mg kg$\left.{ }^{-1}\right)$. The immobility time was evaluated before and after LPS administration ( 6 and $24 \mathrm{hr}$ ). Each value represents the mean of 16 mice performed in two different experimental sets. Data are shown as mean $\pm S E M ; \wedge p<.05$ and ${ }^{\wedge \wedge} p<.01$ versus vehicle + vehicle-treated mice; ${ }^{*} p<.05$ and ${ }^{* *} p<.01$ versus vehicle + LPStreated mice. LPS, lipopolysaccharide

tail. Repeated treatment with B. monnieri extract counteracted the increased of immobility time induced by LPS acute injection in a dosedependent manner. The group treated with the higher dose $\left(200 \mathrm{mg} \mathrm{kg}^{-1}\right)$ reached the value of $59.4 \pm 7.5 \mathrm{~s}$ at $6 \mathrm{hr}$ and 37.8 $\pm 12.2 \mathrm{~s}$ at $24 \mathrm{hr}$ after LPS injection in comparison to the values of 


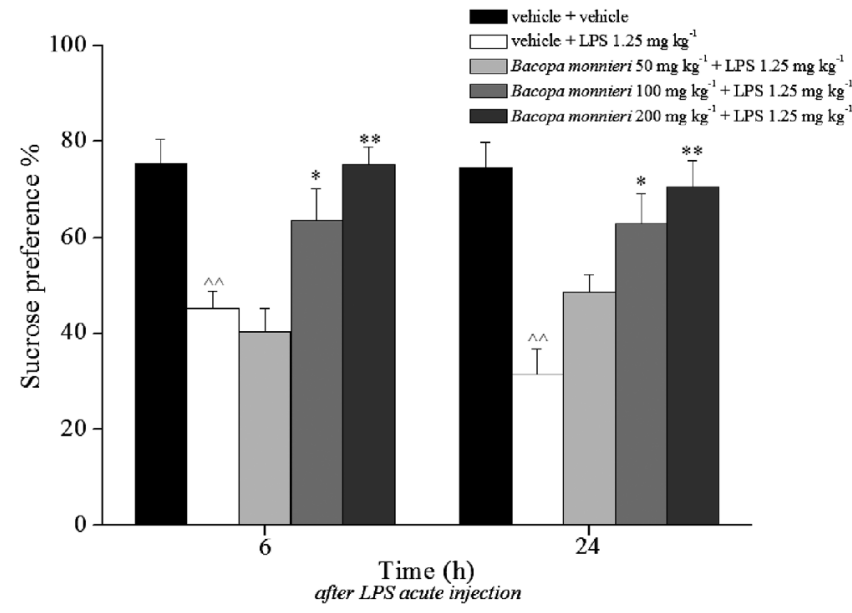

FIGURE 4 Sucrose preference test. Effect of B. monnieri extract on LPS-induced anhedonic-like behavior. B. monnieri (50$200 \mathrm{mg} \mathrm{kg}^{-1}$ ) was p.o. daily administered for 1 week before the acute i.p. injection of LPS $\left(1.25 \mathrm{mg} \mathrm{kg}^{-1}\right)$. The sucrose preference was evaluated after 6 and $24 \mathrm{hr}$ LPS administration. Each value represents the mean of 16 mice performed in two different experimental sets. Data are shown as mean $\pm S E M ; \wedge \wedge<.01$ versus vehicle + vehicletreated mice; ${ }^{*} p<.05$ and ${ }^{* *} p<.01$ versus vehicle + LPS-treated mice. LPS, lipopolysaccharide

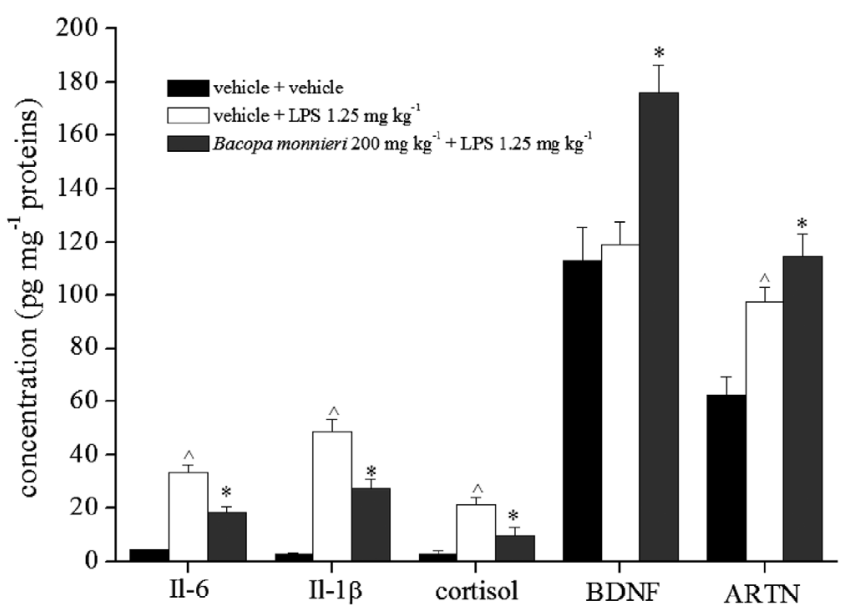

FIGURE 5 Measurements of soluble factors in the prefrontal cortex. Effect of B. monnieri extract on LPS-induced II-6, II-1 $\beta$, cortisol, BDNF, and ARTN alterations. B. monnieri (200 mg kg ${ }^{-1}$ ) was p.o. daily administered for 1 week before the acute i.p. injection of LPS (1.25 mg kg${ }^{-1}$ ). The measurements of II-6, II-1 $\beta$, cortisol, BDNF, and ARTN were performed in the prefrontal cortex $24 \mathrm{hr}$ after LPS administration. Each value represents the mean of 16 mice performed in two different experimental sets. Data are shown as mean $\pm S E M$; ${ }^{\wedge} p<.05$ versus vehicle + vehicle-treated mice; ${ }^{*} p<.05$ versus vehicle + LPS-treated mice. ARTN, artemin; BDNF, brain-derived neurotrophic factor; LPS, lipopolysaccharide

$116.6 \pm 9.8 \mathrm{~s}$ and $87.3 \pm 3.4 \mathrm{~s}$ reached by LPS-treated group (Figure 3). The dose $100 \mathrm{mg} \mathrm{kg}^{-1}$ was less effective while the lower dosage was inactive.

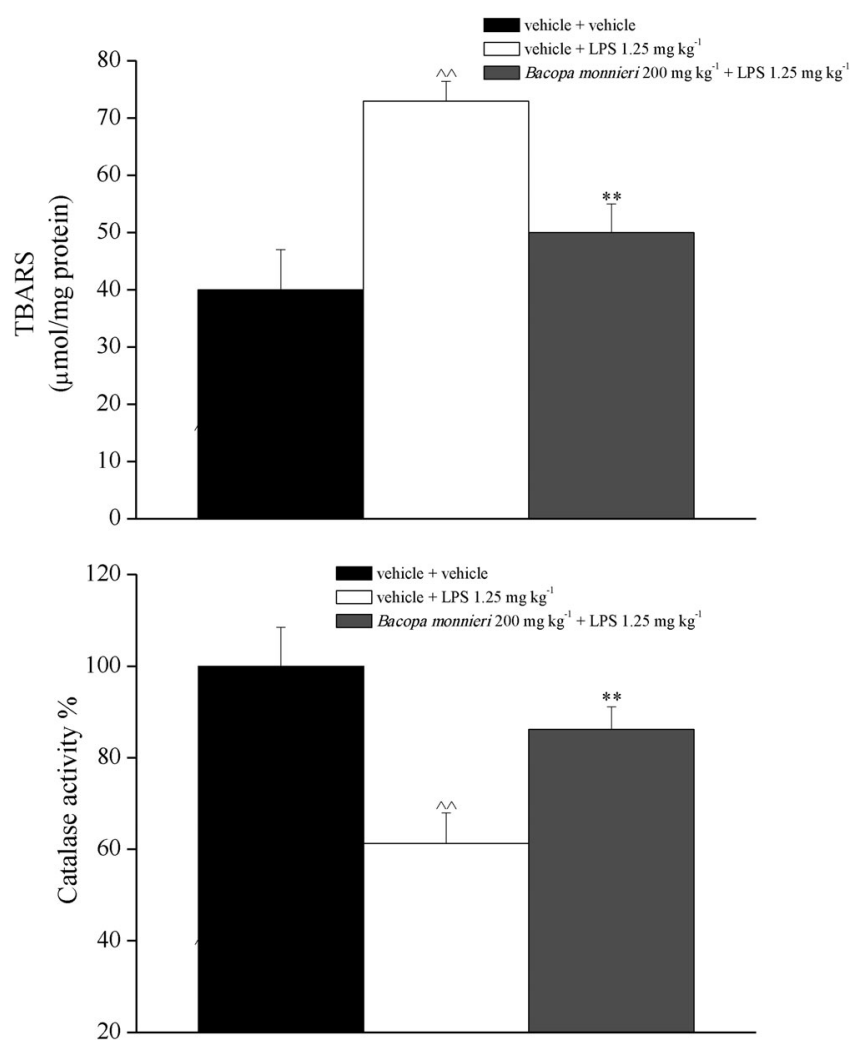

FIGURE 6 Evaluation of TBARS and catalase activity in the prefrontal cortex. Effect of B. monnieri extract on LPS-induced oxidative stress and enzymatic alterations. B. monnieri $\left(200 \mathrm{mg} \mathrm{kg}^{-1}\right)$ was p.o. daily administered for 1 week before the acute i.p. injection of LPS (1.25 mg kg${ }^{-1}$ ). The measurements of (a) TBARS and (b) catalase activity were performed in the prefrontal cortex $24 \mathrm{hr}$ after LPS administration. Each value represents the mean of 16 mice performed in two different experimental sets. Data are shown as mean $\pm S E M ; \wedge p<.01$ versus vehicle + vehicle-treated mice; ${ }^{* *} p<.01$ versus vehicle + LPS-treated mice. LPS, lipopolysaccharide; TBARS, thiobarbituric acid reactive substances

\subsection{3 | Sucrose preference test}

Another anhedonic-like behavior was the reduction of sucrose intake. Six and twenty-four hours after LPS injection, mice in the LPS group showed significantly less preference for sucrose than animals in the control group ( $45.2 \%$ vs $75.4 \%$ at $6 \mathrm{hr}$ and $31.5 \%$ vs $74.7 \%$ at $24 \mathrm{hr}$; Figure 4). B. monnieri extract counteracted this phenomenon at both time points when was administered at the dose of 100 and $200 \mathrm{mg} \mathrm{kg}^{-1}$. The lower dosage was ineffective.

\subsection{4 | Measurement of soluble factors}

Moreover, LPS injection significantly increased the prefrontal cortex concentration of IL-6, IL-1 $\beta$, cortisol, and ARTN with respect to the control group $24 \mathrm{hr}$ after injection. No alteration was recorded for the neurotrophic factor BDNF (Figure 5). B. monnieri $\left(200 \mathrm{mg} \mathrm{kg}^{-1}\right.$ ) 
(a)

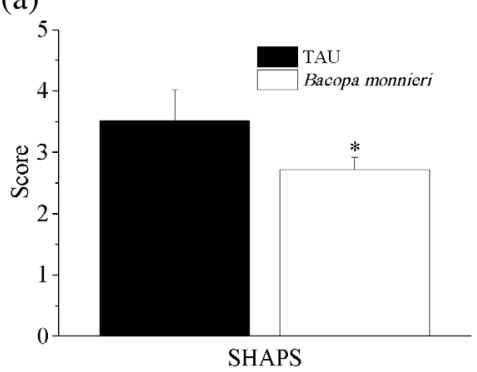

(d)

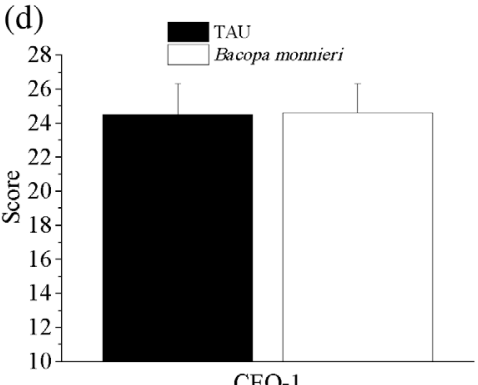

(g)

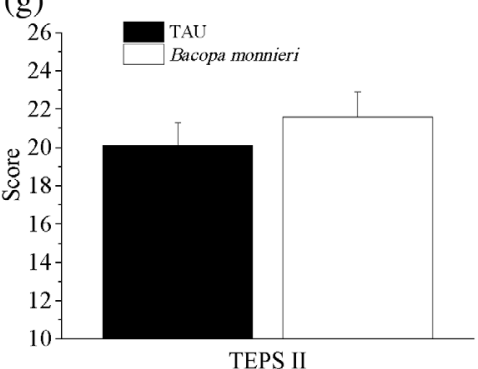

(b)

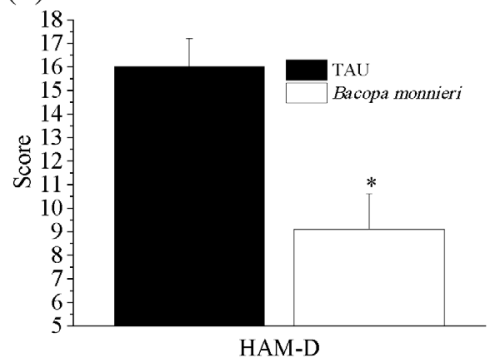

(e)

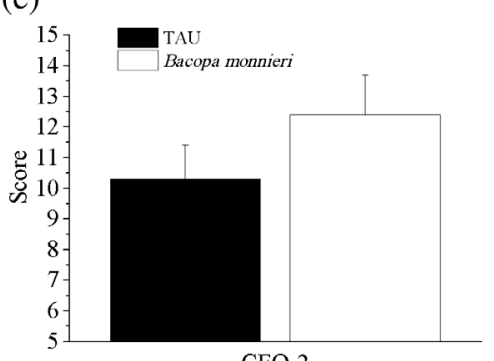

CEQ-2

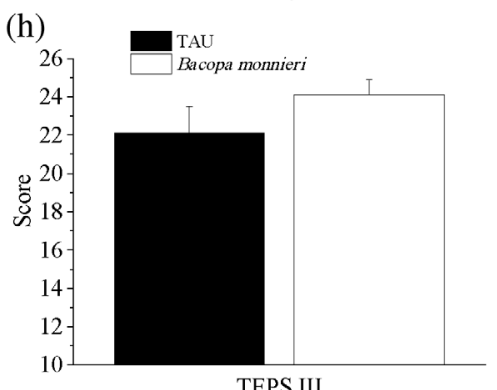

(c)

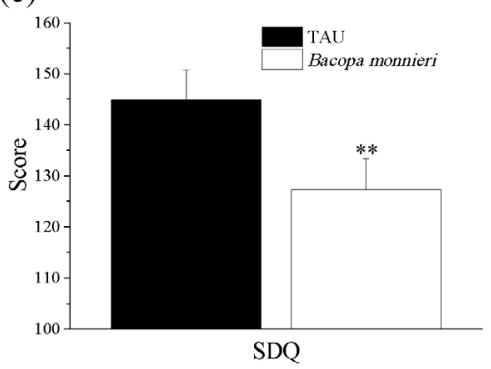

(f)

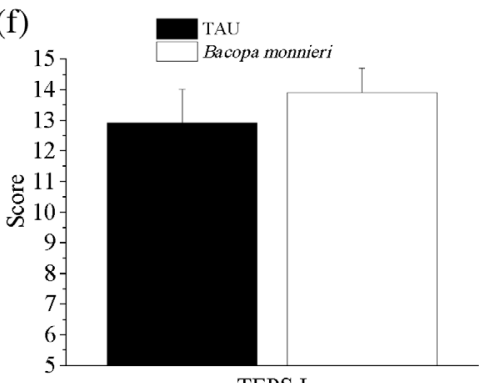

(i)

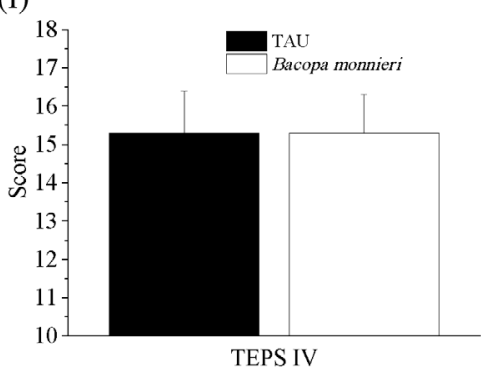

FIGURE 7 Clinical outcomes post-treatment in the group of patients treated with B. monnieri compared to the group treated with citalopram $40 \mathrm{mg}$ (TAU). The first group ( $n=21)$ was treated only with $(40 \mathrm{mg}$ ) citalopram for the follow 4 weeks (TAU); the second group ( $n=19)$ with conventional therapy associated with a nutraceutical supplement consisting of B. monnieri extract titrated at $20 \%$ in bacosides (one capsule $300 \mathrm{mg}$ twice daily). The treatment lasted 4 weeks. Significant differences were measured for HAM-D, SHAPS, and SDQ. ${ }^{*} p<.05$ and ${ }^{* *} p<.01$ versus TAU. HAM-D, Hamilton depression rating scale; SHAPS, Snaith-Hamilton pleasure scale; SDQ, strength and difficulties questionnaire

significantly reduced the LPS-alterations recorded for IL-6, IL-1 $\beta$, cortisol, and ARTN while increased the BDNF value with respect to control and LPS group (Figure 5).

\subsection{5 | Effect of B. monnieri on oxidative stress and catalase activity}

Aimed to evaluate the protective effect of B. monnieri treatment against LPS-induced oxidative damage, we analyzed the lipid peroxidation measuring TBARS levels in the prefrontal cortex. Acute injection of LPS induced an alteration of oxidative stress parameter evaluated as a significantly increased of TBARS levels in comparison to the control groups, B. monnieri treatment restored the unbalance as shown in Figure 6a. Moreover, the lipopolysaccharide administration promoted a decrease in the catalase activity that was fully counteracted by B. monnieri repeated treatment (Figure 6b).

\subsection{Clinical results}

Forty-two patients (Table 1) with anhedonia (SHAPS $\geq 3$ ) and unsatisfactory response to drug therapy (CGI-S $\geq 3$ and CGI-I between 3 and 7) were consequently enrolled, a first group (cases) of 19 patients have been prescribed B. monnieri extract in $300 \mathrm{mg}$ tablets to be taken twice a day, for 4 weeks of treatment, while the second of 23 subjects (controls) with the same characteristics of the cases, which were treated with citalopram $40 \mathrm{mg}$ (TAU).

In Figure 7, the differences in clinical outcomes post-treatment in the group of patients treated with $B$. monnieri compared to the group treated with citalopram $40 \mathrm{mg}$ (TAU) are reported. Significant differences were measured for HAM-D, SHAPS, and SDQ. Raw data of each group pre- and post-treatment are reported in the Table S1. In the group treated with $B$. monnieri, there was no statistically significant correlation between the scores of both of the CEQ subscales and the T1 outcomes, excluding a possible autosuggestion effect by the patient. In the light of these data, the expectation and credibility in 
the treatment of augmentation would not seem to be relevant for the final answer.

\section{4 | DISCUSSION}

Considering our initial hypothesis, this study indicates that $B$. monnieri extract can reduce anhedonia-like behavior in animals and anhedoniarelated symptoms in humans. In mice, the repeated administration of 100 and $200 \mathrm{mg} \mathrm{kg}^{-1}$ extract, dose-dependently, reverted the pathological alterations induced by LPS. Efficacy was highlighted by different paradigms. The forced swimming test induces a "state of despair" that immobilizes the animal in swimming (Carbajal, Ravelo, Molina, Mas, \& Arruzazabala Mde, 2009). This test is considered to have proven reliability and validity (Porsolt et al., 1977) for the analysis of antidepressants. The efficacy of Bacopa in this test was confirmed in the similar tail suspension analysis but also in the sucrose preference test that represents the most indicative analysis for anhedonia evaluation (Liu et al., 2018).

Based on the clinical evidence about the involvement of inflammatory processes in the pathogenesis of depression (Dantzer, O'Connor, Freund, Johnson, \& Kelley, 2008), LPS is one of the most used models of inflammation-associated depression. Behavioral studies in rodents have shown that systemic LPS injection, by a potent activation of the immune response, induces a sickness response, characterized by social withdrawal, fatigue, anorexia, and alterations in sleep patterns and cognition, followed by a behavior similar to clinically relevant symptoms of depression in humans, including anhedonia (Biesmans et al., 2016; Henry et al., 2008; Kang et al., 2011; O'Connor et al., 2009).

The inflammatory response induced by LPS parallels with the production of pro-inflammatory cytokines and the cortisol-dependent depressive-like behavior promoted by chronic stress in laboratory animal (Knapp et al., 2011). Interestingly, treatment with antidepressants, besides normalizing depressive behavior induced by bacterial endotoxin, decreases pro-inflammatory cytokines' level (Castanon, Leonard, Neveu, \& Yirmiya, 2002).

In the present results, we showed enhanced brain levels of the pro-inflammatory cytokines IL-1 $\beta$ and IL6 after LPS treatment. Bacopa extract significantly prevented these alterations suggesting antiinflammatory effects of this product in the CNS. Accordingly, Nemetchek et al. (2017) showed that B. monnieri, as well as Bacoside A, significantly inhibited the release of TNF- $\alpha$ and IL- 6 from LPS-activated microglial cells.

Moreover, it is known that activation of the immune system causes disruption of the HPA axis, a physiological recurrence often observed in depression (Capuron \& Miller, 2011). According to previous results (Goble et al., 2001; Silva \& Madeira, 2012), we found that systemic injection of LPS significantly increased brain cortisol levels. In this condition, the treatment with $B$. monnieri extract significantly prevented the HPA axis dysregulation.

In close relationship with the inflammatory state of the CNS, the altered redox unbalance plays a critical role in the pathophysiology of several neuropsychiatric disorders (Floyd, 1999). Brain tissue is particularly exposed to reactive oxygen species since $20 \%$ of total body oxygen is normally metabolized by brain in the presence of a limited amount of antioxidant strategies (Floyd \& Carney, 1992). In situations where the generation of free radicals exceeds the capacity of antioxidant defense, oxidative stress may lead to membrane degradation, cellular dysfunction, and apoptosis (Ott, Gogvadze, Orrenius, \& Zhivotovsky, 2007). Recent studies reported enhanced concentrations of lipid peroxidation products and alterations of the major antioxidant enzymes in patients with affective disorders, in particular with depression (Floyd, 1999; Ozcan, Gulec, Ozerol, Polat, \& Akyol, 2004; Sarandol et al., 2007); these features were sensitive to antidepressant therapies (Machado-Vieira, Salvadore, Luckenbaugh, Manji, \& Zarate Jr., 2008). B. monnieri treatment was able to restore the oxidative damage occurring in the brain of LPS-treated animals, preventing lipoperoxidation and enhancing the activity of the peroxisome-characteristic detoxifying enzyme catalase.

Interestingly, LPS did not alter the concentration of the neuronal factor BDNF but increased levels of ARTN, a factor of glial origin involved in depression signaling (Di Cesare Mannelli et al., 2011). B. monnieri treatment increased BDNF (considered as a marker of brain functionality increased after antidepressants treatment; Tsai, Hong, \& Liou, 2010) without alter the LPS-induced increase of ARTN suggesting a possible role of these factors, and the consequent cellular plasticity, in the anti-anhedonic effect of the extract. On this base, the extract of B. monnieri was clinically tested. Subjects were evaluated by the MINI (Sheehan et al., 1998) to have a diagnosis standardized according to the DSM 5.0 criteria. After treatment, the group treated with B. monnieri extract showed significant lower value of SHAPS and HAM-D in comparison to TAU group.

Despite the exploratory characteristics of this study (it is an open study with a low number of samples), various corrective measures have been done to validate it. In order to characterize the study sample, a descriptive statistical analysis was performed, in particular for the categorical variables study the chi-square test was used, while the $t$-test was used for the continuous variables. From the first analysis, the two groups were found to be homogeneous for almost all the clinical variables considered, with the exception of CGI3 $(p<.01)$.

To compensate for the lack of a placebo-controlled study, considering the probability of autosuggestion of the patients treated with "natural medicine," it was decided to provide a scale to measure that variable to the base line in order to limit its potential confusing effect. For this purpose, the credibility/expectancy questionnaire (CEQ), developed by Devilly and Borkovec (2000). The credibility of the treatment refers to how credible, convincing and logical the treatment seems to the patient, while the expectation of treatment refers to the actual improvement that the patient feels will be achieved by him/her personally. The CEQ showed a high internal consistency and good test-retest reliability.

It has been postulated that credibility with regard to treatment and expectation may be modifiable during treatment. It is also likely that expectation is a stronger predictor of the treatment outcome than its credibility (Goossens, Vlaeyen, Hidding, Kole-Snijders, \& 
Evers, 2005). A critical issue of the studies in literature is that they have only evaluated the pre-treatment phase immediately prior to the onset of therapy, which means that patients may have difficulty in judging what outcome they might achieve. It is to be specified, therefore, that in our study the CEQ was completed immediately after the patient had obtained the necessary treatment explanations, so that the patient was able to judge the credibility of the treatment and mature one's expectation.

Devilly and Borkovec argued that even if a patient thinks a new treatment is credible, this may differ from what he really feels. The authors hypothesized that expectation may be more related to an emotional aspect than the credibility that is most based on cognitive elements, and therefore may have a higher level of association with the final outcome of treatment, at least in Psychotherapy (Devilly \& Borkovec, 2000). The authors argue that the sub-scale of credibility is more closely related to the logical thinking processes of patients, while the subscale of expectation ("how much is really expected to improve?") is functionally more linked to affective processes similar to those involved in hope or faith. Our study, based on the difference in the magnitude of R2's change for expectation and credibility, did not find clear indications for their hypothesis.

In conclusion, we evaluated a possible effect of a standardized extract of B. monnieri on positive symptoms, especially anhedonia, regardless of categorical diagnosis. The hypothesis of a possible therapeutic application of the botanical on the specific size was initially tested in an animal model that reflected the theoretical construct, targeting specific neurocircuits. The results of the preclinical phase were the basis on which to build the next clinical trial. The significant positive impact of $B$. monnieri treatment in patients was demonstrated. These results reflect the opportunities in applying the RDoC concept to clinical research, and it would of interest to investigate the clinical effect of B. monnieri on the same dimension, anhedonia, in clinical condition different from depression such as negative symptoms in schizophrenia. A harmonization of the models is still needed to effectively isolate the symptoms of "negative valence" in depression and other mental illnesses, and advances their translation to treatment. In particular in the field of Complementary Therapy (CAM), an increasingly evolving field in terms of clinical use but poorly of valid scientific evidence and studies conducted with rigorous scientific criteria according with current neuroscience research. Our work, although it presents all the limits of an exploratory study, may serve as a procedural model for other neurobiological domains, creating the bases for producing a more rigorous and translator clinical research in the field of integrative medicine.

\section{ACKNOWLEDGEMENTS}

This research was supported by the Italian Ministry of Instruction, University, and Research (MIUR) and by the University of Florence.

\section{CONFLICT OF INTEREST}

The authors declare that there are no known conflicts of interest associated with this publication and there has been no significant financial support for this work that could have influenced its outcome.

\section{ORCID}

Lorenzo Di Cesare Mannelli (D) https://orcid.org/0000-0001-83744432

\section{REFERENCES}

Aleman, A., Lincoln, T. M., Bruggeman, R., Melle, I., Arends, J., Arango, C., \& Knegtering, H. (2017). Treatment of negative symptoms: Where do we stand, and where do we go? Schizophrenia Research, 186, 55-62.

Banerjee, R., Hazra, S., Ghosh, A. K., \& Mondal, A. C. (2014). Chronic administration of bacopa monniera increases BDNF protein and mRNA expressions: A study in chronic unpredictable stress induced animal model of depression. Psychiatry Investigation, 11, 297-306.

Benson, S., Downey, L. A., Stoug, C., Wetherell, M., Zangara, A., \& Scholey, A. (2014). An acute, double-blind, placebo-controlled cross-over study of $320 \mathrm{mg}$ and $640 \mathrm{mg}$ doses of Bacopa monnieri (cdri08) on multitasking stress reactivity and mood. Phytotherapy Research, 28, 551-559.

Biesmans, S., Matthews, L. J., Bouwknecht, J. A., De Haes, P., Hellings, N., Meert, T. F., ... Ver Donck, L. (2016). Systematic analysis of the cytokine and anhedonia response to peripheral lipopolysaccharide administration in rats. BioMed Research International, 2016, 9085273.

Biesman, S., Meert, T. F., Bouwknecht, J. A., Acton, P. D., Davoodi, N., De Haes, P., ... Nuydens, R. (2013). Systemic immune activation leads to neuroinflammation and sickness behavior in mice. Mediators of Inflammation, 2013, 271359.

Capuron, L., \& Miller, A. H. (2011). Immune system to brain signaling: Neuropsychopharmacological implications. Pharmacology \& Therapeutics, 130(2), 226-238.

Carbajal, D., Ravelo, Y., Molina, V., Mas, R., \& Arruzazabala Mde, L. (2009). $\mathrm{D}-004$, a lipid extract from royal palm fruit, exhibits antidepressant effects in the forced swim test and the tail suspension test in mice. Pharmacology Biochemistry and Behavior, 92(3), 465-468.

Castanon, N., Leonard, B. E., Neveu, P. J., \& Yirmiya, R. (2002). Effects of antidepressants on cytokine production and actions. Brain, Behavior and Immunity, 16(5), 569-574.

Craske, M. G., Meuret, A. E., Ritz, T., Treanor, M., \& Dour, H. J. (2016). Treatment for anhedonia: A neuroscience driven approach. Depression and Anxiety, 33(10), 927-938.

Dantzer, R., O'Connor, J. C., Freund, G. G., Johnson, R. W., \& Kelley, K. W. (2008). From inflammation to sickness and depression: When the immune system subjugates the brain. Nature Reviews Neuroscience, 9 (1), 46-56.

Devilly, G. J., \& Borkovec, T. D. (2000). Psychometric properties of the credibility/expectancy questionnaire. Journal of Behavior Therapy Experimental Psychiatry, 31(2), 73-86.

Di Cesare Mannelli, L., Vivoli, E., Salvicchi, A., Schiavone, N., Koverech, A., Messano, M., ... Ghelardini, C. (2011). Antidepressant-like effect of artemin in mice: A mechanism for acetyl-L-carnitine activity on depression. Psychopharmacology, 218(2), 347-356.

Floyd, R. A., \& Carney, J. M. (1992). Free radical damage to protein and DNA: Mechanism involved and relevant observations on brain undergoing oxidative stress. Annals of Neurology, 32, 522-527.

Floyd, R. A. (1999). Antioxidants, oxidative stress, and degenerative neurological disorders. Proceedings of the Society for Experimental Biology, 222, 236-245.

Goble, K. H., Bain, Z. A., Padow, V. A., Lui, P., Klein, Z. A., \& Romeo, R. D. (2001). Pubertal-related changes in hypothalamic-pituitary-adrenal axis reactivity and cytokine secretion in response to an immunological stressor. Journal of Neuroendocrinology, 23(2), 129-135.

Goossens, M. E., Vlaeyen, J. W., Hidding, A., Kole-Snijders, A., \& Evers, S. M. (2005). Treatment expectancy affects the outcome of cognitivebehavioral interventions in chronic pain. Clinical Journal of Pain, 21, 18-26. 
Hamilton, M. (1960). A rating scale for depression. Journal of Neurology, Neurosurgery, and Psychiatry, 23, 56-62.

Hasler, G., Drevets, W. C., Manji, H. K., \& Charney, D. S. (2004). Discovering endophenotypes for major depression. Neuropsychopharmacology, 29(10), 1765-1781.

Hazra, S., Kumar, S., Saha, G. K., \& Mondal, A. C. (2017). Reversion of BDNF, Akt and CREB in hippocampus of chronic unpredictable stress induced rats: Effects of phytochemical, Bacopa monnieri. Psychiatry Investigation, 14(1), 74-80.

Henry, C. J., Huang, Y., Wynne, A., Hanke, M., Himler, J., Bailey, M. T., ... Godbout, J. P. (2008). Mynocyline attenuates lipopolysaccharide (LPS)-induced neuroinflammation, sickness behavior, and anhedonia. Journal of Neuroinflammation, 5, 15.

ICH, International Conference on Harmonisation. (2005). Q2 (A)Validation of analytical procedures: Text and methodology (pp. 1-13). Geneva: International Conference on Harmonization.

Insel, T. R. (2014). The NIMH Research Domain Criteria (RDoC) project: Precision medicine for psychiatry. American Journal of Psychiatry, 171 (4), 395-397.

Kang, A., Hao, H., Zheng, X., Liang, Y., Xie, Y., Xie, T., ... Wang, G. (2011). Peripheral anti-inflammatory effects explain the ginsenosides paradox between poor brain distribution and anti-depression efficacy. Journal of Neuroinflammation, 8, 100.

Knapp, D. J., Whitman, B. A., Wills, T. A., Angel, R. A., Overstreet, D. H., Criswell, H. E., ... Breese, G. R. (2011). Cytokine involvement in stress may depend on corticotrophin releasing factor to sensitize ethanol withdrawal anxiety. Brain, Behavior and Immunity, 1, S146-S154.

Kumar, S., \& Mondal, A. C. (2016). Neuroprotective, Neurotrophic and anti-oxidative role of Bacopa monnieri on CUS induced model of depression in rat. Neurochemical Research, 41(11), 3083-3094.

Liu, M. Y., Yin, C. Y., Zhu, L. J., Zhu, X. H., Xu, C., Luo, C. X., ... Zhou, Q. G. (2018). Sucrose preference test for measurement of stress-induced anhedonia in mice. Nature Protocols, 13, 1686-1698.

Machado-Vieira, R., Salvadore, G., Luckenbaugh, D. A., Manji, H. K., \& Zarate, C. A., Jr. (2008). Rapid onset of antidepressant action: A new paradigm in the research and treatment of major depressive disorder. Journal of Clinical Psychiatry, 69, 946-958.

McGrath, J. C., \& Lilley, E. (2015). Implementing guidelines on reporting research using animals (ARRIVE etc.): New requirements for publication in BJP. British Journal of Pharmacology, 172(13), 3189-3193.

Negi, K. S., Singh, Y. D., \& Kushwaha, K. P. (2000). Clinical evaluation of memory enhancing properties of memory plus in children with attention deficit hyperactivity disorder. Indian Journal of Psychiatry, 42, 42-50.

Nemetchek, M. D., Stierle, A. A., Stierle, D. B., \& Lurie, D. I. (2017). The Ayurvedic plant Bacopa monnieri inhibits inflammatory pathways in the brain. Journal of Ethnopharmacology, 197, 92-100.

O'Connor, J. C., Lawson, M. A., André, C., Moreau, M., Lestage, J., Castanon, N., ... Dantzer, R. (2009). Lipopolysaccharide-induced depressive-like behavior is mediated by indoleamine 2,3-dioxygenase activation in mice. Molecular Psychiatry, 14(5), 511-522.

Ott, M., Gogvadze, V., Orrenius, S., \& Zhivotovsky, B. (2007). Mitochondria, oxidative stress and cell death. Apoptosis, 12, 913-922.

Ozcan, M. E., Gulec, M., Ozerol, E., Polat, R., \& Akyol, O. (2004). Antioxidant enzyme activities and oxidative stress in affective disorders. International Clinical Psychopharmacology, 19, 89-95.

Pan, H., Mukhopadhyay, P., Rajesh, V., Mukhopadhyay, B., Gao, B., Hasko, G., \& Pacher, P. (2009). Cannabidiol attenuates cisplatin-induced nephrotoxicity by decreasing oxidative/nitrosative stress, inflammation, and cell death. Journal of Pharmacology and Experimental Therapeutics, $3,708-714$
Pettorruso, M., Spagnolo, P. A., Leggio, L., Janiri, L., Di Giannantonio, M., Gallimberti, L., ... Martinotti, G. (2018). Repetitive transcranial magnetic stimulation of the left dorsolateral prefrontal cortex may improve symptoms of anhedonia in individuals with cocaine use disorder: A pilot study. Brain Stimulation, 11(5), 1195-1197.

Pettorruso, M., Di Giannantonio, M., De Risio, L., Martinotti, G., \& Koob, G. F. (2019). A light in the darkness: Repetitive transcranial magnetic stimulation (rTMS) to treat the hedonic dysregulation of addiction. Journal of Addiction Medicine. https://doi.org/10.1097/ADM. 0000000000000575

Pham, H. T. N., Phan, S. V., Tran, H. N., Phi, X. T., Le, X. T., Nguyen, K. M., ... Matsumoto, K. (2019). Bacopa monnieri (L.) ameliorates cognitive deficits caused in a trimethyltin-induced neurotoxicity model mice. Biological and Pharmaceutical Bulletin, 42(8), 1384-1393.

Porsolt, R. D., Le Pichon, M., \& Jalfre, M. E. (1977). Depression: A new animal model sensitive to antidepressant treatments. Nature, 266 730-732.

Sarandol, A., Sarandol, E., Eker, S. S., Erdinc, S., Vatansever, E., \& Kirli, S. (2007). Major depressive disorder is accompanied with oxidative stress: Short-term antidepressant treatment does not alter oxidativeantioxidative systems. Human Psychopharmacology, 22, 67-73.

Sheehan, D. V., Lecrubier, Y., Sheehan, K. H., Amorim, P., Janavas, J., Weiller, H., ... Dunbar, G. C. (1998). The Mini-International Neuropsychiatric (interview (M.I.N.I): The development and validation of a structured diagnostic psychiatric interview for DSM-IV and ICD-10. The Journal of Clinical Psychiatry, 59(Suppl 20), 22-33.

Sheikh, N., Ahmad, A., Siripurapu, K. B., Kuchichotla, V. K., Singh, S., \& Palit, G. (2007). Bacopa Monnier's effect on stress induced changes in corticosterone plasma and brain monoamines in rats. Journal of Ethnopharmacology, 111(3), 671-676.

Silva, S. M., \& Madeira, M. D. (2012). Effects of chronic alcohol consumption and withdrawal on the response of the male and female hypothalamic-pituitary-adrenal axis to acute immune stress. Brain Research, 1444, 27-37.

Snaith, R. P., Hamilton, M., Morley, S., Humayan, A., Hargreaves, D., \& Trigwell, P. (1995). A scale for the assessment of hedonic tone: The Snaith-Hamilton pleasure scale. British Journal of Psychiatry, 167, 99-103.

Tsai, S. J., Hong, C. J., \& Liou, Y. J. (2010). Effects of BDNF polymorphisms on antidepressant action. Psychiatry Investigation, 7(4), 236-242.

Treadway, M. T., \& Zald, D. H. (2011). Reconsidering anhedonia in depression: Lessons from translational neuroscience. Neuroscience \& Biobehavioral Reviews, 35(3), 537-555.

Zanardelli, M., Micheli, L., Cinci, L., Failli, P., Ghelardini, C., \& Di Cesare Mannelli, L. (2014). Oxaliplatin neurotoxicity involves peroxisome alterations. PPAR $\gamma$ agonism as preventive pharmacological approach. PLOS ONE, 9(7), e102758.

\section{SUPPORTING INFORMATION}

Additional supporting information may be found online in the Supporting Information section at the end of this article.

How to cite this article: Micheli L, Spitoni S, Di Cesare Mannelli L, Bilia AR, Ghelardini C, Pallanti S. Bacopa monnieri as augmentation therapy in the treatment of anhedonia, preclinical and clinical evaluation. Phytotherapy Research. 2020;34:2331-2340. https://doi.org/10.1002/ptr.6684 